\title{
Growth, photosynthesis and podophyllotoxin accumulation of Dysosma versipellis in response to a light gradient and conservation implications
}

\author{
ZHAO YunPeng ${ }^{1}$, GONG HuaDong ${ }^{1}$, LU WeiQiang ${ }^{1}$, JIANG WeiMei ${ }^{1}$, ZHOU XiaoLong $^{2}$ \& \\ FU ChengXin ${ }^{1 *}$ \\ ${ }^{1}$ Key Laboratory of Conservation Biology for Endangered Wildlife of the Ministry of Education, College of Life Sciences, Zhejiang University, \\ Hangzhou 310058, China; \\ ${ }^{2}$ Institute of Chinese Materia Medica, Pan'an 321004, China
}

Received February 28, 2011; accepted May 4, 2011

\begin{abstract}
Dysosma versipellis (Hance) M. Cheng ex Ying (Berberidaceae) is a rare and vulnerable, perennial herb endemic to China with pharmaceutical significance. Increasing habitat loss and over-exploitation of the plant has severely affected the plant's in situ conservation, necessitating ex situ conservation and commercial cultivation. The light regime is a critical environmental factor contributing to successful ex situ conservation via efficient production of biomass and secondary metabolites. We investigated the responses of 2-year-old $D$. versipellis plants to a light gradient in terms of leaf morphology, growth, biomass production, photosynthesis and podophyllotoxin (PTOX) accumulation. D. versipellis responded to the light gradient in terms of plant height, leaf size, leaf photosynthesis and PTOX accumulation. Plants demonstrated optimal vegetative growth, photosynthetic capability, and PTOX production, under a light intensity equal to $30 \%$ full sunlight. Half full sunlight led to the lowest production of PTOX despite having no obviously different effects on growth. Full sunlight was able to cause leaf death in mid-summer (July), although these plantlets did not significantly differ in growth or biomass production before July and new leaves emerged in August. $D$. versipellis is therefore proposed to be a shade-tolerant plant, well adapted to variations in irradiance. Irradiance of $10 \%-50 \%$, particularly ca. $30 \%$ full sunlight is strongly recommended for ex situ conservation and commercial cultivation.
\end{abstract}

Dysosma versipellis, light response, photosynthesis, podophyllotoxin accumulation, ex situ conservation

Citation: Zhao Y P, Gong H D, Lu W Q, et al. Growth, photosynthesis and podophyllotoxin accumulation of Dysosma versipellis in response to a light gradient and conservation implications. Chinese Sci Bull, 2011, 56: 2570-2575, doi: 10.1007/s11434-011-4565-1

Ex situ conservation has played an increasingly important role in stemming loss of biodiversity [1]. A simulation of a natural habitat can guarantee $e x$ situ survival of conserved plants. Light regime is a critical environmental factor affecting plant growth, distribution, and yield [2,3]. To achieve the ultimate goal of biodiversity conservation, commercial plantations of wild economic plants, including medicinal plants, will be essential. Thus, the yield of a desired natural product is the key for industrial scale production. Light variation can be a significant factor affecting

*Corresponding author (email: cxfu@zju.edu.cn) secondary metabolism in plants as well as plant growth [4]. Some surveys have supported the effect of the light regime on plant secondary metabolism [5-7]. An optimal light intensity is, therefore, a question needing exploration for successful ex situ conservation or efficient commercial plantation of each endangered plant.

Dysosma versipellis (Hance) M. Cheng ex Ying (Berberidaceae) is a rare and vulnerable, perennial herb endemic to China [8]. A total of 30 populations are known to inhabit disjunct stands of warm-temperate, deciduous, montane forest across central and eastern China [9]. The plant's rhizome has long been applied for the treatment of snake bites, 
parotitis, and rheumatism, in traditional medicine [10]. In recent decades $D$. versipellis has attracted the interest of the pharmaceutical industry due to the presence of podophyllotoxin (PTOX), a lignan used as a leading compound in the synthesis of several popular anti-tumor drugs [11,12]. Excessive medicinal exploitation coupled with natural habitat loss has rendered $D$. versipellis at a higher risk of extinction, necessitating immediate protection $[13,14]$. To conserve this endangered species, we established successful systems for both ex situ conservation and pre-commercial cultivation [15-17]. However, its physioecology, including the effects of light intensity on its growth and PTOX production, remains poorly evaluated.

The general biosynthetic pathway of PTOX has been outlined previously [18]. Deoxypodophyllotoxin has been thought to be the common precursor of both PTOX and $\beta$-peltatin; these can be further synthesized into PTOXglucoside and $\beta$-peltatin-glucoside, respectively. Despite a body of research into PTOX production at the cellular level in vitro and relating to levels of specific compounds in the plant tissues in vivo, no assessment has yet been performed on PTOX dynamics in vivo in light of PTOX biosynthesis.

We therefore addressed the following questions: (i) How do $D$. versipellis plants respond to a light gradient in terms of morphology, biomass yield, and photosynthesis? and (ii) What is the response of PTOX accumulation to a light gradient in vivo? Our results will improve the understanding of plant responses to variation in irradiance to facilitate optimal light intensity for both ex situ conservation and commercial cultivation.

\section{Materials and methods}

\subsection{Study station and plant materials}

The field experiment was conducted at Linfeng Farm of Medicinal Plants, Pan'an County, Zhejiang Province, China $\left(120^{\circ} 32-56^{\prime \prime} \mathrm{E}, 29^{\circ} 1-25^{\prime \prime} \mathrm{N}, 835 \mathrm{~m}\right.$ a.s.l.) in a typical subtropical zone with mild seasonal temperature variation and plentiful precipitation. The climate is mainly characterized by average annual air temperature: $13.9-17.4^{\circ} \mathrm{C}$, extremely low temperature in January: $2^{\circ} \mathrm{C}$, extremely high temperature in July: $28.8^{\circ} \mathrm{C}$, average annual precipitation: $1409.8-1527.8 \mathrm{~mm}$, and average precipitation in July and August: 208.3-246.5 mm.

Plantlets of $D$. versipellis produced via tissue culture were transplanted to the Medicinal Plant Farm in April, 2008, and survivals with a uniform size (ca. $10 \mathrm{~cm}$ in height measured in August 2008) were marked and introduced to experimental blocks on April 1, 2009. D. versipellis is a typical understory herb in habitats with a light intensity of $10 \%-40 \%$ (occasionally up to $58 \%$ ) full sunlight. It can survive full sunlight after introduction 4 natural habitats (data not shown). Accordingly, four irradiance levels (10\%, $30 \%, 50 \%$ and $100 \%$ sunlight) were simulated using poly- propylene shading cloth. Each treatment included 7 plants. Based on our phenological observations in the Botanical Garden of Zhejiang University, these perennial plants with 5-7 rhizomatous nodules introduced from the wild produced shoots in early March, bloomed in early April, subsequently set fruit, senesced in August, and perished in late September (data not shown). The plants regenerated via tissue culture, however, presented no flowers during the first 3 years, and displayed longer annual vegetative growth, growing until October. Plant materials were harvested on September 1, prior to leaf senescence, for analysis.

\subsection{Leaf photosynthesis, plant morphology, growth and biomass analyses}

Gas exchange measurements were made on the centers of fully expanded, healthy leaves using a portable LI-6400 photosynthesis system (LI-COR, Lincoln, USA) in open system mode, during the morning (07:00-11:00) of a sunny day (August 28, 2009). Three replications were performed for each light gradient. Net photosynthetic rate $\left(P_{\mathrm{n}}\right)-$ photosynthetic photon flux density (PPFD) response curves were generated based on the assumption of natural conditions (approximately $380 \mu \mathrm{mol} \mathrm{mol}{ }^{-1} \mathrm{CO}_{2}$, relative humidity of $40 \%-60 \%, 500 \mu \mathrm{mol} \mathrm{s}{ }^{-1}$ air flow, and temperature of $\left.(30 \pm 3)^{\circ} \mathrm{C}\right)$ at irradiance gradients of $1800,1600,1400$, $1000,600,300,100,60,30,10$ and $0 \mu \mathrm{mol} \mathrm{m} \mathrm{m}^{-2} \mathrm{~s}^{-1}$, using a built-in LED-B light source.

Plant height, leaf blade diameter, and petiole diameter were measured on all individuals each month during the studied growing season. We compared the data generated on August 21 (closest to when photosynthesis was measured - August 28) with that generated when the plant materials were harvested (September 1). Finally, 3 of the 7 individuals in each treatment were harvested, separated into leaves (blades plus petioles), rhizomes and roots. All samples were dried at $40^{\circ} \mathrm{C}$ for $48 \mathrm{~h}$, and then weighed.

\subsection{Lignan determination}

The dried rhizomes were ground using a MM400 ball mill (Retsch, Germany), and ultrasonically extracted with methanol $(0.1 \mathrm{~g}: 2 \mathrm{~mL})$ for $30 \mathrm{~min}$. The extraction was centrifuged at $10000 \mathrm{r} / \mathrm{min}$ for $10 \mathrm{~min}$ preceding filtering of the supernatant through a $0.22-\mu \mathrm{m}$ membrane following a procedure modified from that previously described [19]. All solutions were analyzed using a Varian Prostar 210 HPLC system, consisting of a ProStar 335 diode array detector, a manual injector, a column oven, and a data system (Galaxie Workstation). Chromatographic separation was performed on an Agilent HC C18 column (250 $\mathrm{mm} \times 4.6 \mathrm{~mm}, 5 \mu \mathrm{m})$ using a gradient of methanol (A) and $0.01 \%$ aqueous phosphoric acid (B). The gradient program was designed as follows: 0-15 $\mathrm{min}, 15 \%-45 \%$ A; $15-45 \mathrm{~min}, 45 \% \mathrm{~A} ; 45-55$ min, $45 \%-70 \%$ A; 55-75 min, 70\%-75\% A; with flow rate of $1 \mathrm{~mL} \mathrm{~min}^{-1}$. Column temperature was maintained at $35^{\circ} \mathrm{C}$, 
and the detection wavelength was set at $254 \mathrm{~nm}$. Five lignans were qualified and quantified with corresponding reference compounds: PTOX (purity $\geqslant 99 \%$, National Institute for Food and Drug Control), deoxypodophyllotoxin (purity $\geqslant 98 \%$, provided by Professor Xuan Tian, Lanzhou University, China), 4'-podophyllotoxin-glucoside (purity $\geqslant 95 \%$ ), $\beta$-peltatin (purity $\geqslant 94 \%$ ) and $\beta$-peltatin-glucoside (purity $\geqslant 94 \%$ ). We prepared the latter three from the rhizomes of $D$. versipellis.

\subsection{Statistical analyses}

Significance of difference of each parameter among treatments was evaluated by one-way ANOVA followed by a LSD post hoc test with a significance level of 0.05 , using the SPSS software (version 16).

\section{Results}

\subsection{Growth, morphology and biomass allocation}

Light significantly affected the leaf morphology of $D$. versipellis. Leaves were severely damaged under full sunlight in mid-summer (July), although they grew vigorously in the spring (from April to June). Following leaf death from over-irradiance, new leaves emerged in August. Plants growing under the other 3 light levels displayed vigorous, green leaves; those under 30\% sunlight appeared relatively darker in color. Plant height, leaf blade diameter and petiole diameter differed significantly between the 4 light treatments. Generally, plants were shorter with smaller leaves as sunlight intensity increased (Figure 1). There were no significant differences in petiole diameter with increasing light gradient except that plants under 50\% sunlight had thinner petioles. All plants produced similar total biomass (no statistical differences), as well as similar allocation patterns (Figure 2). D. versipellis allocated an average of $85 \%$ of its biomass to underground organs (rhizomes plus roots) and the remaining $15 \%$ to the aerial plant parts.

\subsection{Leaf photosynthesis}

Regardless of the irradiance treatment, $P_{\mathrm{n}}$ values rose rapidly as PPFD increased to $400 \mu \mathrm{mol} \mathrm{m} \mathrm{m}^{-2} \mathrm{~s}^{-1}$, and then increased slowly to a maximum followed by a general leveling off until the greatest PPFD of $1800 \mu \mathrm{mol} \mathrm{m}^{-2} \mathrm{~s}^{-1}$ was reached (Figure 3). The light compensation point (LCP) of plants in full sunlight $\left(72.48 \pm 26.07 \mu \mathrm{mol} \mathrm{m} \mathrm{m}^{-2} \mathrm{~s}^{-1}\right)$ was significantly higher than of those under $50 \%(24.60 \pm 9.95 \mu \mathrm{mol}$ $\left.\mathrm{m}^{-2} \mathrm{~s}^{-1}\right), 30 \%\left(8.46 \pm 4.00 \mu \mathrm{mol} \mathrm{m}{ }^{-2} \mathrm{~s}^{-1}\right)$ and $10 \%(12.01$ $\left.\pm 2.95 \mu \mathrm{mol} \mathrm{m}^{-2} \mathrm{~s}^{-1}\right)$ sunlight. All test plants displayed similar light saturation points (LSPs) of $600 \mu \mathrm{mol} \mathrm{m} \mathrm{m}^{-2} \mathrm{~s}^{-1}$. Plants under $30 \%$ sunlight treatment showed the highest maximum $P_{\mathrm{n}}\left(P_{\max }\right)$ while those under full sunlight exhibited the lowest, and those under either $10 \%$ or $50 \%$ sunlight gave intermediate values.

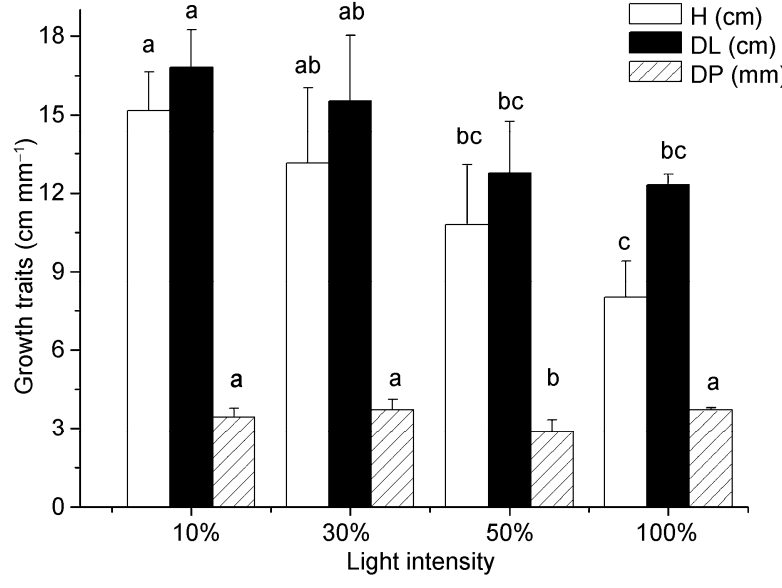

Figure 1 Comparisons of vegetative growth traits of $D$. versipellis under different light conditions. H, plant height; DL, leaf blade diameter; DP, petiole diameter. Lowercase letters indicate significant differences $(\alpha=0.05)$.

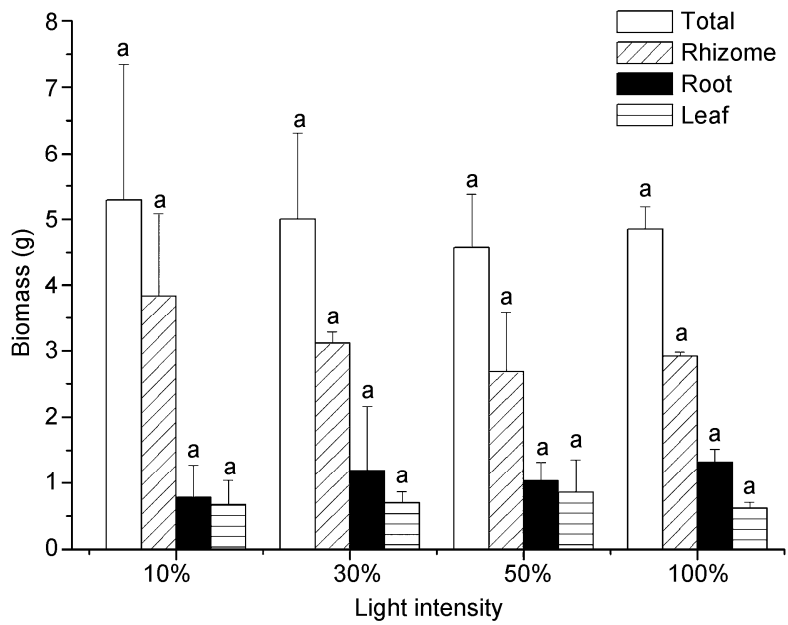

Figure 2 Production and allocation of vegetative biomass of $D$. versipellis under different light conditions. Total, total biomass; rhizome, biomass in rhizomes; root, biomass in roots; leaf, biomass in leaves. Lowercase letters indicate significant differences $(\alpha=0.05)$.

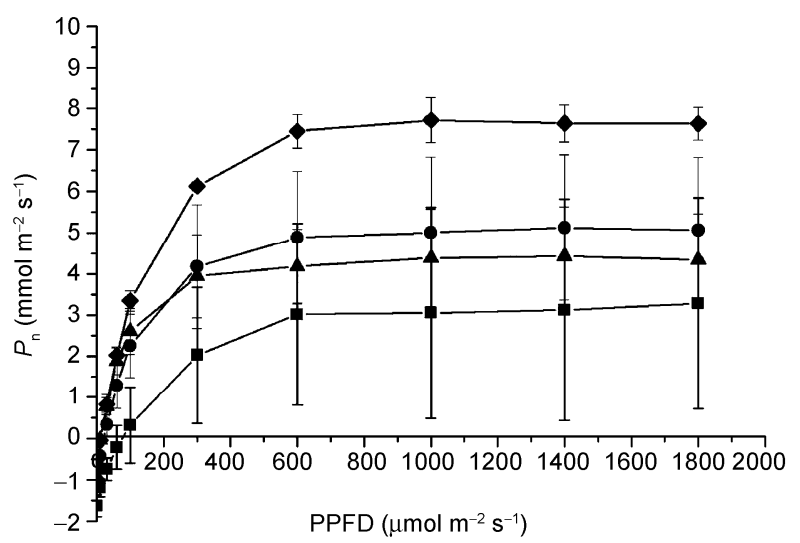

Figure 3 Photosynthetic light response curves of $D$. versipellis under different light conditions. Irradiance rate: $10 \%(\boldsymbol{\Delta}), 30 \%(\bullet), 50 \%(\bullet)$, and $100 \%(\mathbf{\square})$. 
Both LCP and $P_{\max }$ values varied significantly $(P<0.05)$ across the light gradient. The greatest value of $P_{\max }$ and the lowest value of LCP were observed in plants under $30 \%$ sunlight.

\subsection{Lignan dynamics}

Levels of 5 analyzed lignans varied significantly in the rhizomes of $D$. versipellis. Deoxypodophyllotoxin was the most abundant $\left(16.00 \pm 3.70 \mu \mathrm{mol} \mathrm{g}{ }^{-1}\right) ; 4.2$ times the level of PTOX and 12.7 times the level of $\beta$-peltatin (Table 1). PTOX levels were higher than those of $\beta$-peltatin. Both of these two aglycones were at far lower levels than their corresponding glycosides, leading to significantly higher levels of total glycoside. Total levels of PTOX and its glycoside were higher than those of $\beta$-peltatin and its glycoside, and the ratio of $\beta$-peltatin-glucoside to $\beta$-peltatin was significantly higher than that of PTOX-glucoside to PTOX.

PTOX and its derivatives varied remarkably with increasing light gradient. PTOX was at higher levels under $30 \%$ and full sunlight, than under $10 \%$ and $50 \%$ sunlight. $\beta$-peltatin only exhibited a rise under full sunlight (Table 1). Their common precursor, deoxypodophyllotoxin, maintained relatively stable levels except for a significant decrease under $50 \%$ sunlight. PTOX-glucoside showed the same trend as deoxypodophyllotoxin. $\beta$-peltatin-glucoside was the most variable lignan analyzed; it was at its highest concentrations under middle irradiance (30\% and 50\% sunlight) and was significantly reduced with either an increase or a decrease in irradiance. $\beta$-peltatin-glucoside was also the only metabolite showing increased levels under $50 \%$ sunlight. Total glycoside levels appeared highest under $30 \%$ sunlight, and fell with either an increase or a decrease in irradiance. The ratio of $\beta$-peltatin-glucoside to $\beta$-peltatin was quite close to the ratio of PTOX-glucoside to PTOX under extremely high $(100 \%)$ or low $(10 \%)$ irradiance, whereas the former was ca. 4.5 times the latter under middle irradiance (30\% and 50\%).

\section{Discussion}

Irradiance levels greatly affected plant growth. As expected, the tested $D$. versipellis plants growing under decreasing irradiance produced increasingly larger leaves and taller plants. This is consistent with the trend observed in many other shade-tolerant plant species, especially in Podophyllum peltatum [20], a North American Berberidaceae species that is famous for its PTOX. We found that larger leaves, however, did not possess greater leaf mass, and consequently the light gradient did not influence total leaf mass in addition to total mass.

D. versipellis, however, appeared to respond to changes in irradiance by changing its photosynthetic capacity. Plants exhibited the strongest photosynthetic capability in terms of $P_{\mathrm{n}}$ under $30 \%$ sunlight, and showed significant reductions under irradiance of both flanking spectra. This parallels the patterns found in another shade-tolerant plant, Tetrastigma hemsleyanum [21]. The trends in the light compensation point (LCP) coincided with those in $P_{\mathrm{n}}$ in $D$. versipellis, and were in contrast to those in $T$. hemsleyanum, in which LCPs were reduced under full and 50\% sunlight. The LCP of our studied plant under $10 \%$ sunlight was similar to that under $30 \%$ sunlight with smallest value; this may indicate shade adaptation [22]. Plants acclimated to a high light environment tend to display increased respiration and LCP [22]. This could explain why the highest LCP of the emerged leaves of $D$. versipellis was under full sunlight, suggesting acclimation to strong sunlight. LCP and LSP are considered effective indicators of the light requirements of plants, and sciophytes generally exhibit lower values than heliophytes [23]. D. versipellis demonstrated smaller LCPs (8.46-72.48 $\mu \mathrm{mol} \mathrm{m} \mathrm{s}^{-1}$ ) and greater LSPs (ca. $600 \mu \mathrm{mol} \mathrm{m} \mathrm{s}^{-2}$ ) than Sinopodophyllum hexandrum (synonyms: Podophyllum

Table 1 Levels of podophyllotoxin and its derivatives in rhizomes of $D$. versipellis under different light conditions $\left(\mu \mathrm{mol}^{-1}\right)$

\begin{tabular}{|c|c|c|c|c|c|}
\hline Irradiance rate & $10 \%$ & $30 \%$ & $50 \%$ & $100 \%$ & Mean $^{\text {a) }}$ \\
\hline$\beta$-peltatin-glucoside & $4.27 \pm 0.40 \mathrm{~b}$ & $7.66 \pm 0.79 \mathrm{a}$ & $7.91 \pm 0.13 \mathrm{a}$ & $1.75 \pm 0.60 \mathrm{c}$ & $5.40 \pm 2.70 b c$ \\
\hline$\beta$-peltatin & $1.07 \pm 0.11 \mathrm{~b}$ & $1.21 \pm 0.03 \mathrm{~b}$ & $1.17 \pm 0.07 \mathrm{~b}$ & $1.58 \pm 0.06 \mathrm{a}$ & $1.26 \pm 0.21 d$ \\
\hline Podophyllotoxin & $2.33 \pm 0.21 \mathrm{~b}$ & $4.88 \pm 0.80 \mathrm{a}$ & $2.98 \pm 0.24 \mathrm{~b}$ & $5.14 \pm 0.05 \mathrm{a}$ & $3.84 \pm 1.31 c$ \\
\hline 4'-podophyllotoxin-glucoside & $7.59 \pm 0.56 \mathrm{a}$ & $6.72 \pm 1.35 \mathrm{a}$ & $4.57 \pm 0.64 \mathrm{~b}$ & $8.15 \pm 1.41 \mathrm{a}$ & $6.76 \pm 1.69 b$ \\
\hline Total & $33.93 \pm 6.74 \mathrm{a}$ & $37.32 \pm 3.73 \mathrm{a}$ & $28.51 \pm 2.31 \mathrm{~b}$ & $33.23 \pm 4.54 \mathrm{a}$ & \\
\hline Total glucosides & $11.86 \pm 0.52 \mathrm{~b}$ & $14.38 \pm 0.86 \mathrm{a}$ & $12.48 \pm 0.53 \mathrm{~b}$ & $9.91 \pm 0.82 \mathrm{c}$ & $12.16 \pm 1.77 a$ \\
\hline$\beta$-peltatin and its glucoside & $5.35 \pm 0.30 \mathrm{~b}$ & $8.87 \pm 0.82 \mathrm{a}$ & $9.08 \pm 0.07 \mathrm{a}$ & $3.33 \pm 0.65 \mathrm{c}$ & $6.66 \pm 2.57 b$ \\
\hline PTOX and its glucoside & $9.92 \pm 0.51 \mathrm{~b}$ & $11.60 \pm 1.52 \mathrm{ab}$ & $7.55 \pm 0.79 \mathrm{c}$ & $13.30 \pm 1.38 \mathrm{a}$ & $10.59 \pm 2.42 a$ \\
\hline Ratio of peltatin-glucoside to peltatin & $4.03 \pm 0.81 \mathrm{~b}$ & $6.35 \pm 0.48 \mathrm{a}$ & $6.81 \pm 0.52 \mathrm{a}$ & $1.10 \pm 0.34 \mathrm{c}$ & $4.57 \pm 2.41 a$ \\
\hline Ratio of PTOX-glucoside to PTOX & $3.27 \pm 0.44 \mathrm{a}$ & $1.40 \pm 0.36 \mathrm{~b}$ & $1.53 \pm 0.19 \mathrm{~b}$ & $1.59 \pm 0.28 \mathrm{~b}$ & $1.95 \pm 0.85 b$ \\
\hline
\end{tabular}

a) Italic lowercase letters indicate significance of vertical comparison, and non-italic indicate significance of horizontal comparison ( $\alpha=0.05)$. 
hexandrum and $P$. emodi); the latter showed LCPs of $19.30-104.00$ and LSPs of 211.00-452.00 $\mu \mathrm{mol} \mathrm{m} \mathrm{m}^{-2} \mathrm{~s}^{-1}$ [24]. Because $S$. hexandrum prefers sunlight and tolerates shade, D. versipellis seems to have adapted to greater variation in irradiance. This could explain our observations of irradiance in the natural habitat ranging from $10 \%$ to $58 \%$ sunlight, and also the survival of $D$. versipellis under full sunlight (data not shown). However, the highest value of $P_{\max }$ and vigorous growth under $30 \%$ sunlight indicated its preference for shade. This is also consistent with the most frequent light intensity in natural habitats of about $30 \%$ sunlight (data not shown).

Similarly to its relative, $P$. peltatum [20], and other reported plant species [6,7], D. versipellis responded prominently to the light gradient in terms of its production of secondary metabolites of the five lignans including PTOX. Deoxypodophyllotoxin, the common precursor of both PTOX and $\beta$-peltatin, showed a high and stable concentration with increasing light gradient. It seemed that the allocation of deoxypodophyllotoxin differed between the two shunt pathways, and was further affected by changes in light intensity. The shunt pathway of PTOX significantly preceded that of $\beta$-peltatin in terms of the total concentration of aglycone and the corresponding glycoside. The concentration peak in the former pathway was produced under either $30 \%$ or $50 \%$ sunlight, in contrast to that in the latter pathway, produced at $100 \%$ or under $30 \%$ sunlight. Generally, $30 \%$ sunlight benefited high production in both pathways. Interestingly, the dynamic trend in the total glycosides (PTOX-glucoside and $\beta$-peltatin-glucoside) paralleled that of photosynthetic capacity $\left(P_{\mathrm{n}}\right)$ (Figure 3 and Table 1). However, the two shunt pathways showed inconsistent responses. It appeared that the $\beta$-peltatin pathway was more sensitive to changes in light intensity because fluctuation was noticeable in the levels of $\beta$-peltatin or its glycoside; their ratio indicating the degree of glycosylation. Increased glycosylation may be one of the consequences of increasing photosynthesis and assimilation. On the contrary, $\beta$-peltatinglucoside was strongly deglycosylated when photoinhibited. PTOX glycosylation remained relatively stable except for a significant response to the weakest irradiance (10\% sunlight).

\section{Conclusion}

$D$. versipellis responded to a light gradient in terms of plant height, leaf size, leaf photosynthesis and PTOX accumulation. The plant demonstrated optimal vegetative growth and photosynthetic capability as well as PTOX production under a light intensity of $30 \%$ full sunlight. Half full sunlight led to the lowest production of PTOX despite no remarkably different effects on growth. Full sunlight was observed to lead to leaf death in 2-year-old plantlets in mid-summer
(July), although these plantlets differed insignificantly in growth and biomass production before July and new leaves emerged in August. D. versipellis is thus proposed to be a shade-tolerant plant with well adapted to variation in irradiance. Irradiance of $10 \%-50 \%$, particularly ca. $30 \%$ full sunlight is strongly recommended for ex situ conservation and commercial cultivation.

We are grateful to Professor De'an Jiang and Mr. Yue Chen for their help with measurement and data analysis of the photosynthesis section. We also thank Mr. Xinhang Jiang and Dr. Zhican Wang for their technical assistance with LC-MS analysis. Our further thanks go to Professor Xuan Tian for providing deoxypodophyllotoxin, and to Dr. Shihua Wu for help with phytochemical isolation and characterization. This work was supported by the National Key Project of Scientific and Technical Supporting Programs of China (2008BAC39B05), the National Basic Research Program of China (2007CB411600), and the Zhejiang Provincial Major Project of Science and Technology (2006C13077).

1 Ali N S, Trivedi C. Botanic gardens and climate change: A review of scientific activities at the Royal Botanic Gardens, Kew. Biodivers Conserv, 2011, 20: 295-307

2 Rothstein D E, Zak D R. Photosynthetic adaptation and acclimation to exploit seasonal periods of direct irradiance in three temperate, deciduous-forest herbs. Funct Ecol, 2001, 15: 722-731

3 Poorter L. Resource capture and use by tropical forest tree seedlings and their consequences for competition. In: Burslem D, Pinard M, Hartley S, eds. Biotic Interactions in the Tropics: Their Role in the Maintenance of Species Diversity. Cambridge: Cambridge University Press, 2005. 35-64

4 Theis N, Lerdau M. The evolution of function in plant secondary metabolites. Int J Plant Sci, 2003, 164 (Suppl): S93-S102

5 Coelho G G, Rachwal M F G, Dedecek N A, et al. Effect of light intensity on methylxanthine contents of Ilex paraguariensis A St. Hil. Biochem Syst Ecol, 2007, 35: 75-80

6 Wang M L, Jiang Y S, Wei J Q, et al. Effects of irradiance on growth, photosynthetic characteristics, and artemisinin content of Artemisia annua L. Photosynthetica, 2008, 46: 17-20

7 Cai Z Q, Wang W H, Yang J, et al. Growth, photosynthesis and root reserpine concentrations of two Rauvolfia species in response to a light gradient. Ind Crop Prod, 2009, 30: 220-226

8 Wang S, Xie Y. China Species Red List (Vol. 1). Beijing: Higher Education Press, 2004. 324

9 Ying T S, Zhang Y L, Boufford D E. The Endemic Genera of Seed Plants of China. Beijing: Science Press, 1993

10 Jiangsu New Medical College. Dictionary of Chinese Traditional Medicine. Shanghai: Shanghai Science and Technology Press, 1986

11 Adlercreutz H. Lignans and human health. Crit Rev Clin Lab Sci, 2007, 44: 483-525

12 Gordaliza M, Garcia P A, Del Corral J M, et al. Podophyllotoxin: Distribution, sources, applications and new cytotoxic derivatives. Toxicon, 2004, 44: 441-459

13 Qiu Y X, Zhou X W, Fu C X, et al. A preliminary study of genetic variation in the endangered, Chinese endemic species Dysosma versipellis (Berberidaceae). Bot Bull Acad Sin, 2005, 46: 61-69

14 Qiu Y X, Guan B C, Fu C X, et al. Did glacials and/or interglacials promote allopatric incipient speciation in East Asian temperate plants? Phylogeographic and coalescent analyses on refugial isolation and divergence in Dysosma versipellis. Mol Phylogenet Evol, 2009, 51: 281-293

15 Pan Q. Study of techniques of propagation in vitro and analysis of chemical constituent on Dysosma versipellis (Berberidaceae). Dissertation for Master Degree. Hangzhou: Zhejiang University, 2004 
16 Wang X Y. Study of propagation in vitro and analysis of chemical constituent of Dysosma versipellis. Dissertation for Master Degree. Hangzhou: Zhejiang University, 2006

17 Shen Y Y. Tissue culture-cultivation and podophyllotoxin determination of medicine plants, Dysosma versipellis and D. pleiantha (Berberidaceae). Dissertation for Master Degree. Hangzhou: Zhejiang University, 2010

18 Yousefzadi M, Sharifi M, Behmanesh M, et al. Podophyllotoxin: Current approaches to its biotechnological production and future challenges. Eng Life Sci, 2010, 10: 281-292

19 Zhou Y, Jiang S, Ding L, et al. Chemical fingerprinting of medicinal plants "gui-jiu" by LC-ESI multiple-stage MS. Chromatographia, 2008, 68: 781-789

20 Cushman K E, Maqbool M, Lata H, et al. Podophyllotoxin content and yield of American mayapple leaves in sun and shade. HortScience, 2005, 40: 60-63

21 Dai Y J, Shen Z G, Liu Y, et al. Effects of shade treatments on the photosynthetic capacity, chlorophyll fluorescence, and chlorophyll content of Tetrastigma hemsleyanum Diels et Gilg. Environ Exp Bot, 2009, 65: 177-182

22 Lambers H, Chapin F S, Pons T L. Plant Physiological Ecology. New York: Springer Verlag, 1998

23 Larcher W. Physiological Plant Ecology: Ecophysiology and Stress Physiology of Functional Groups. Heidelberg: Springer Verlag, 2003, 513

24 Shang H L, Li F M, Lin Y, et al. Photosynthetic characteristics of Sinopodophyllum hexandrum from different distribution areas in china. Acta Bot Bor-occid Sin, 2008, 28: 1440-1447

Open Access This article is distributed under the terms of the Creative Commons Attribution License which permits any use, distribution, and reproduction in any medium, provided the original author(s) and source are credited. 